\title{
Orbit determination and time synchronization for BDS-3 satellites with raw inter-satellite link ranging observations
}

\author{
Rengui Ruan ${ }^{1,2,3^{*}} \mathbb{0}$, Xiaolin Jia ${ }^{1,2}$, Laiping Feng ${ }^{1,2}$, Jun Zhu ${ }^{4,5}$, Zongbo Huyan ${ }^{4,5}$, Jie Lii, ${ }^{4,5}$ and Ziqing Wei ${ }^{1,2}$
}

\begin{abstract}
To provide competitive global positioning and timing services under the condition that monitoring stations are confined to Chinese territory, inter-satellite link (ISL) technology is used by the third-generation BeiDou Navigation Satellite System (BDS-3). The ISL, together with the dual one-way links between satellites and anchor stations, may enable autonomous navigation for BDS-3. In this paper, we propose a general observation model for orbit determination (OD) and time synchronization (TS) directly using non-simultaneous observations, such as raw ISL pseudoranges. With the proposed model, satellite orbits, clocks, and hardware delay biases of ISL equipment can be determined simultaneously by jointly processing inter-satellite one-way pseudorange data and observation data from ground monitoring stations. Moreover, autonomous OD and TS are also achievable with one-way pseudorange data from anchor stations and satellites. Data from eight BDS-3 satellites, two anchor stations, and seven monitoring stations located in China were collected to validate the proposed method. It is shown that by jointly processing data from the ISL and seven monitoring stations, the RMS of overlap orbit differences in radial direction is $0.019 \mathrm{~m}$, the overlap clock difference (95\%) is $0.185 \mathrm{~ns}$, and the stability of the estimated hardware delay biases for each satellite is greater than $0.5 \mathrm{~ns}$. Compared with the results obtained with the seven stations, the improvements of orbits in radial direction and clocks are $95.7 \%$ and $90.5 \%$, respectively. When the hardware delay biases are fixed to predetermined values, the accuracies of orbits and clocks are further improved. By jointly processing pseudoranges from the satellites and the two anchor stations, the RMS of overlap orbit differences is $0.017 \mathrm{~m}$ in the radial direction, and the overlap clock difference (95\%) is $0.037 \mathrm{~ns}$. It has also been demonstrated that under the condition of one-way ranging links, the accuracies of orbits and clocks obtained by the above two modes are still significantly better than those obtained by using the data from the monitoring stations alone.
\end{abstract}

Keywords: BDS-3, Inter-satellite link, Anchor station, Orbit determination, Time synchronization, Hardware delay bias

\section{Introduction}

To enhance orbit determination (OD) and time synchronization (TS) under the condition of regional monitoring stations, the third-generation BeiDou Navigation Satellite System (BDS-3) employs inter-satellite link (ISL) technology which also enable autonomous navigation. The feasibility and performance of the ISL

\footnotetext{
*Correspondence: rrg2002me@163.com

${ }^{1}$ Xi'an Research Institute of Surveying and Mapping, Xi'an 710054, China Full list of author information is available at the end of the article
}

in BDS-3 have been verified with the in-orbit validation satellites [1-7]. Recent findings show that with the ISL ranging data, the radial orbit accuracy of $10 \mathrm{~cm}$ is achievable [2].

Phased array antennas on BDS-3 satellites receive and transmit ISL signals in Ka-band and control the beam pointing of the signal. The Ka-band signal with frequencies more than 10 times the L-band signal would provide greater communication bandwidth and make the ISL antennas smaller in size, which make it preferred for ISL. The ISL of BDS-3 satellites operates in a time-division multiple access (TDMA) mode, i.e., at any time (slot), 
one satellite can only communicate and perform ranging measurement with another satellite according to the preset link planning. Pseudorange measurements observed at one satellite to others are generated in different time slots.

In existing methods of OD or TS for Global Navigation Satellite System (GNSS) satellites with ISL ranging data, the raw non-simultaneously observed pseudoranges are not directly used. Instead, in a typical approach originally proposed for autonomous OD and TS [8], observations are derived at specified times, and then the derived clock measurement (DCM) and the derived ephemeris measurement (DEM) are formed to separate geometry information and clock information [8-11]. The DEMs are used for OD alone $[2,5-9,12-14]$ or together with other data such as the monitoring station data [3] and the inter-satellite orientation observations [15], while the DCMs are used for TS [4]. Therefore, OD and TS are performed independently. In another approach, OD and TS are performed simultaneously by directly processing the one-way derived ISL observations together with the monitoring station data [16-18]. An advantage of the latter method is that the hardware delay biases of ISL equipment of each satellite can be estimated together with satellite orbits and clocks. However, the latter has only been used in simulation studies at present, and there have been no reports on the application for BDS-3.

A method to directly process raw one-way ISL pseudoranges for OD and TS of BDS-3 satellites was proposed by Ruan $[19,20]$. In this paper, the principal of this method will be presented in detail, and its feasibility will be demonstrated with two typical modes for OD and TS: (1) jointly processing the ISL pseudorange data and the L-band pseudorange and carrier phase data from monitoring stations, and (2) jointly processing the Ka-band ISL pseudorange data between satellites and the Ka-band pseudorange data between satellites and anchor stations. The anchor station refers to a station equipped with ISL equipment and can perform communication and ranging with BDS-3 satellites, just as the satellites do. Besides, it will be demonstrated that the proposed OD and TS method is achievable even under the condition of oneway ranging links between satellites (and between satellites and anchor stations).

\section{Methodologies}

\section{Observation equation for ISL pseudoranges}

The ISL pseudorange observations of satellite $j$ observed by satellite $i$ at time $t^{i, j}$ are expressed as

$$
\begin{aligned}
P^{i, j}\left(t^{i, j}\right)= & \left|\boldsymbol{R}^{i}\left(t^{i, j}\right)-\boldsymbol{R}^{j}\left(t^{i, j}-\tau^{i, j}\right)\right| \\
& +\delta^{i}\left(t^{i, j}\right)-\delta^{j}\left(t^{i, j}-\tau^{i, j}\right)+\Delta^{j}+\beta^{i}+\omega,
\end{aligned}
$$

where $\tau^{i, j}$ is the propagation time of ISL signal from satellite $j$ to satellite $i ; \boldsymbol{R}^{i}$ and $\boldsymbol{R}^{j}$ are the instantaneous positions of satellite $i$ and $j$ at the receiving and transmitting times, respectively; $\delta^{i}$ and $\delta^{j}$ are the clock offsets of satellite $i$ and $j$, respectively; $\Delta^{j}$ and $\beta^{i}$ are the transmitting and receiving hardware delays of satellite $j$ and $i$, respectively, considered to be stable in time, and $\omega$ is the measurement noise [2, 17]. Equation (1) omits terms such as relativistic delay of signal propagation and satellite antenna phase center offsets.

Equation (1) can also be used for the pseudorange observations between BDS-3 satellites and anchor stations, when the troposphere delay and ionosphere delay are taken into account. Since the BDS-3 ISL runs in Ka-band, pseudorange observations between satellites or between satellites and anchor stations are uniformly referred to as Ka-pseudoranges.

\section{Observation equation for pseudoranges and phases at monitoring stations}

Without losing generality, ionosphere-free carrier phase $L_{r}^{i}$ and pseudorange $P_{r}^{i}$ of satellite $i$ observed by station (receiver) $r$ at the time $t_{k}$ can be expressed as

$$
\begin{aligned}
& L_{r}^{i}\left(t_{k}\right)=\left|\boldsymbol{R}_{r}\left(t_{k}\right)-\boldsymbol{R}^{i}\left(t_{k}-\tau_{r}^{i}\right)\right|+\theta_{r, k}-\theta_{k}^{i}+T_{r}^{i}+a_{r}^{i}+\varepsilon \\
& P_{r}^{i}\left(t_{k}\right)=\left|\boldsymbol{R}_{r}\left(t_{k}\right)-\boldsymbol{R}^{i}\left(t_{k}-\tau_{r}^{i}\right)\right|+\theta_{r, k}-\theta_{k}^{i}+T_{r}^{i}+\xi
\end{aligned}
$$

where $k$ is the observation epoch; $\tau_{r}^{i}$ is the propagation time of signal from satellite $i$ to station $r ; \boldsymbol{R}_{r}$ is the instantaneous position of the station at the receiving time; $\theta_{r, k}=\delta_{r, k}+b_{r}$ and $\theta_{k}^{i}=\delta_{k}^{i}-b^{i}$ are the estimable clock parameters for the station and the satellite, i.e., superposition of the clock offset $\left(\delta_{r, k}\right.$ and $\left.\delta_{k}^{i}\right)$ and the ionosphere free combination of hardware group delay $\left(b_{r}\right.$ and $\left.b^{i}\right)$; $a_{r}^{i}$ is the ionosphere-free phase ambiguity parameter, including the hardware phase and group delays; $T_{r}^{i}$ is the troposphere delay; $\varepsilon$ and $\xi$ are measuring errors of carrier phase and pseudorange measurements, respectively [21]. Equation (2) omits the correction terms of relativistic delay, phase wind-up effect and antenna phase centre offsets of the satellite and the station.

\section{General observation equation for non-simultaneous observations}

Pseudoranges and carrier phases [Eq. (2)] and Ka-pseudoranges [Eq. (1)] are radio measurements that contains clock offsets of the signal transmitting and receiving devices. In order to directly process the non-simultaneous observation data, such as the raw Ka-pseudorange observations of ISL, a general observation model is proposed in this section.

Continuous time is divided into discrete non-overlapping time slots with length of $w$. In any time slot $k$, the 
behavior of clocks on transmitting or receiving devices can be modeled with a polynomial, thus, clock offsets at any time $t$ within time slot $k$ are, according to Ruan [19],

$$
\delta^{i}(t)=\delta_{k}^{i}+\dot{\delta}_{k}^{i} \cdot\left(t-t_{w, k}\right)+\ddot{\delta}_{k}^{i} \cdot\left(t-t_{w, k}\right)^{2}+\cdots,
$$

where $t_{w, k}$ is the selected reference time for the time slot $k$ and is preferably at the middle point of the time slot. $\delta_{k}^{i}$ is the zero-order coefficient of the polynomial, i.e., clock bias at $t_{w, k} ; \dot{\delta}_{k}^{i}$ and $\ddot{\delta}_{k}^{i}$ are first and second order coefficients, i.e., drift and acceleration of the clock, respectively. Terms of higher order are omitted in Eq. (3) (usually terms higher than the second order can be safely ignored). In practice, accurate modeling of long-term changes of clocks using polynomials is challenging. In order to control the error of the clock polynomial models, the length of time slots should not be too long, depending on the frequency instability of an individual clock. For example, in a short time period, such as a few minutes, the clock of a GNSS satellite can usually be modeled using a linear polynomial, with adequate precision.

In this way, a radio measurement $O^{r, e}$ for a transmitting device $e$ observed at $t^{r, e}$ by the receiving device $r$ can be expressed as, according to Ruan [19],

$$
\begin{aligned}
O^{r, e}\left(t^{r, e}\right)= & \rho^{r, e}+\left[\delta_{k}^{r}+\dot{\delta}_{k}^{r} \cdot\left(\Delta t^{r, e}\right)+\cdots\right] \\
& -\left[\delta_{k}^{e}+\dot{\delta}_{k}^{e} \cdot\left(\Delta t^{r, e}-\tau^{r, e}\right)+\cdots\right]+\omega_{o},
\end{aligned}
$$

where $t^{r, e}$ is within the time slot $k, \Delta t_{k}^{r, e}=t^{r, e}-t_{w, k}$ is the difference between observing time and the reference time $t_{w, k}, \rho^{r, e}$ is the distance from a transmitting antenna to a receiving antenna, and $\omega_{0}$ is the measuring error. The above formula does not include other factors that may affect the observation.

Specifically, if clock offsets are modeled with linear polynomials, the observation equation, Eq. (1), for Ka-pseudoranges observed at time $t^{i, j}$, which is within the time slot $k$, can be rewritten as

$$
\begin{aligned}
P^{i, j}\left(t^{i, j}\right)= & \left|\boldsymbol{R}^{i}\left(t^{i, j}\right)-\boldsymbol{R}^{j}\left(t^{i, j}-\tau^{i, j}\right)\right| \\
& +\left[\delta_{k}^{i}+\dot{\delta}_{k}^{i} \cdot\left(\Delta t_{k}^{i, j}\right)\right]-\left[\delta_{k}^{j}+\dot{\delta}_{k}^{j} \cdot\left(\Delta t_{k}^{i, j}-\tau^{i, j}\right)\right] . \\
& +\Delta^{j}+\beta^{i}+\omega
\end{aligned}
$$

Thus, for any satellite-pair, all Ka-pseudoranges within the same time slot share the same clock parameters, although they are observed at different times. For example, a satellite's clock parameters are first and second order coefficients in Eq. (5). The non-simultaneously observed Ka-pseudoranges of satellites (and anchor stations) can be directly processed separately or jointly with other tracking data, estimation of orbits, clocks, and even hardware delay biases can be achieved simultaneously.
In practice, the drift of satellite clocks can usually be predicted with a relatively high accuracy; therefore, the first order coefficients of clock models in Eq. (5) can be replaced by predicted values. For example, the uncertainty of predicted clock drift of a BDS satellite is less than $1 \times 10^{-13} \mathrm{~s} / \mathrm{s}$ [2], and if the linear variation of the clock is corrected with the predicted clock drift, the resulting modeling error is less than $2 \mathrm{~mm}$ for time slots with length less than $1 \mathrm{~min}$. In extreme cases without predicted clock drift, clock variation during short durations can even be directly ignored. Therefore, with predicted clock drift or by ignoring the clock variation, Eq. (5) is simplified to

$$
P^{i, j}\left(t^{i, j}\right)=\left|\boldsymbol{R}^{i}\left(t^{i, j}\right)-\boldsymbol{R}^{j}\left(t^{i, j}-\tau^{i, j}\right)\right|+\delta_{k}^{i}-\delta_{k}^{j}+\Delta^{j}+\beta^{i}+\bar{\omega},
$$

where $\bar{\omega}$ is the sum of measuring error and additional errors caused by predicted clock drift or ignoring the clock drift.

The conventional observation model for pseudorange and phase observations observed at monitoring stations, such as in Eq. (2), can be regarded as a special case of Eq. (4) because each time slot contains only one epoch; thus, the model for the receiver clock is simplified with only the zero-order term. For any satellite, the time difference of signal transmitting corresponding to observations simultaneously observed by different stations at the same epoch is very small (i.e., much less than $1 \mathrm{~s}$ ), so the variation of satellite clock can also be completely ignored.

\section{Orbit determination and time synchronization of BDS-3 satellites}

For OD and TS of the BDS-3 satellites, there are several types of tracking data that can be used, including pseudoranges and carrier phases observed at monitoring stations, mainly located within Chinese territory, Kapseudoranges observed at satellites and anchor stations, and two-way satellite time frequency transferring data between stations and satellites. For the proposed general observation model, the orbits and clocks of BDS-3 satellites can theoretically be determined by independently processing any type of tracking data or jointly processing several data types. This paper focuses on two modes for OD and TS:

- Mode-1 Jointly processing ISL Ka-pseudoranges observed from satellites and observation data from monitoring stations;

- Mode-2 Processing Ka-pseudoranges observed from both satellites and anchor stations.

In Mode-1, satellite orbits, clocks, and the hardware delay biases of each satellite are determined 
simultaneously [17]. This is a significant mode for BDS-3 and may be the best OD and TS mode, as it is an important and self-consistent method, which can resolve the inconsistency of hardware delays of the ISL equipment and the navigation signal generation equipment. In order to construct the joint observation model with both ISL data and the data from monitoring stations, the simplest method is to adopt the observation epoch time $t_{k}$ of the monitoring station as the reference time of specific time slots; i.e., $t_{w, k}=t_{k}$. Thus, the zero-order coefficient of the clock polynomial is exactly the clock offset $\theta_{k}^{i}$ at the time $t_{k}$. Omitting detailed derivation, joint observation equation with ISL Ka-pseudoranges and ionosphere-free pseudoranges, and phases at time slot $k$ centered on its reference time $t_{k}$ is expressed as follows: where $a$ represents anchor stations and $T_{a}^{i}$ is the troposphere delay in Ka-pseudoranges between satellites and anchor stations. Ka-biases are not present in Eq. (8) because they are linearly correlated with the clock parameters when Ka-pseudoranges are used alone and must be calibrated with known values, e.g., estimates obtained by Mode- 1 , to achieve an unbiased clock solution for positioning and timing applications.

\section{Validations \\ Data set and experimental scheme}

To validate the proposed approach, the Ka-pseudorange data from eight BDS-3 satellites (B19-B22, B27-B30), two anchor stations (A01 and A02), and the pseudorange and carrier phase data from B1I and B3I frequency from

$$
\begin{aligned}
L_{r}^{i}\left(t_{k}\right)= & \left|\boldsymbol{R}_{r}\left(t_{k}\right)-\boldsymbol{R}^{i}\left(t_{k}-\tau_{r}^{i}\right)\right|+\theta_{r, k}-\theta_{k}^{i}+T_{r}^{i}+b_{r}^{i}+\varepsilon \\
P_{r}^{i}\left(t_{k}\right)= & \left|\boldsymbol{R}_{r}\left(t_{k}\right)-\boldsymbol{R}^{i}\left(t_{k}-\tau_{r}^{i}\right)\right|+\theta_{r, k}-\theta_{k}^{i}+T_{r}^{i}+\xi \\
P^{i, j}\left(t^{i, j}\right)= & \left|\boldsymbol{R}^{i}\left(t^{i, j}\right)-\boldsymbol{R}^{j}\left(t^{i, j}-\tau^{i, j}\right)\right| \\
& +\left[\theta_{k}^{i}+\dot{\theta}_{k}^{i} \cdot \Delta t_{k}^{i, j}\right]-\left[\theta_{k}^{j}+\dot{\theta}_{k}^{j} \cdot\left(\Delta t_{k}^{i, j}-\tau^{i, j}\right)\right]+\Delta_{b}^{j}+\beta_{b}^{i}+\bar{\omega}
\end{aligned}
$$

where $-\frac{w}{2} \leq t^{i, j}-t_{k}<\frac{w}{2}, \quad \dot{\theta}_{k}^{*}$ is the clock drift; $\Delta_{b}^{*}=\Delta^{*}-b^{*}$ and $\beta_{b}^{*}=\beta^{*}+b^{*}$ are the transmitting and receiving hardware delay biases for ISL equipment on satellite $*(=i, j)[17]$, referred to as transmitting and receiving Ka-biases, respectively, in this context. The definition of Ka-biases and the estimation method are presented in Ruan et al. [16, 17]. In Eq. (7), the observation time of the Ka-pseudoranges is usually different from the sampling time of the L-band pseudorange and phase data, but they share the same clock parameters. After modeling and linearization, unknown parameters such as satellite orbit, clock parameters, and Ka-biases are solved simultaneously, e.g., with the least square method.

Mode- 2 uses Ka-pseudoranges observed from both satellites and anchor stations, and is usually referred to as (centralized) autonomous OD and TS [2, 10]. The observation equations are seven monitoring stations of the International GNSS Monitoring and Assessment System (iGMAS) during days $152-163$ of 2018 were collected. The distribution of the iGMAS stations used in the experiment is shown in Fig. 1.

Table 1 shows the signal transmission and reception between satellites or between satellites and anchor stations during the data arc. It can be seen that each satellite has a bidirectional inter-satellite measurement link with 5-6 satellites, and each satellite has a bidirectional measurement link with the two anchor stations, except satellite B30.

With the above data, validation of OD and TS was performed by the following experiments:

- EXP1: Only data from the iGMAS stations were used.

$$
\begin{aligned}
P^{i, j}\left(t^{i, j}\right)= & \left|\boldsymbol{R}^{i}\left(t^{i, j}\right)-\boldsymbol{R}^{j}\left(t^{i, j}-\tau^{i, j}\right)\right| \\
& +\left[\theta_{k}^{i}+\dot{\theta}_{k}^{i} \cdot\left(\Delta t_{k}^{i, j}\right)\right]-\left[\theta_{k}^{j}+\dot{\theta}_{k}^{j} \cdot\left(\Delta t_{k}^{i, j}-\tau^{i, j}\right)\right]+\bar{\omega} \\
P^{i, a}\left(t^{i, a}\right)= & \left|\boldsymbol{R}^{i}\left(t^{i, a}\right)-\boldsymbol{R}^{a}\left(t^{i, a}-\tau^{i, a}\right)\right| \\
& +\left[\theta_{k}^{i}+\dot{\theta}_{k}^{i} \cdot\left(\Delta t_{k}^{i, a}\right)\right]-\left[\theta_{k}^{a}+\dot{\theta}_{k}^{a} \cdot\left(\Delta t_{k}^{i, a}-\tau^{i, a}\right)\right]+T_{a}^{i}+\bar{\omega}
\end{aligned}
$$




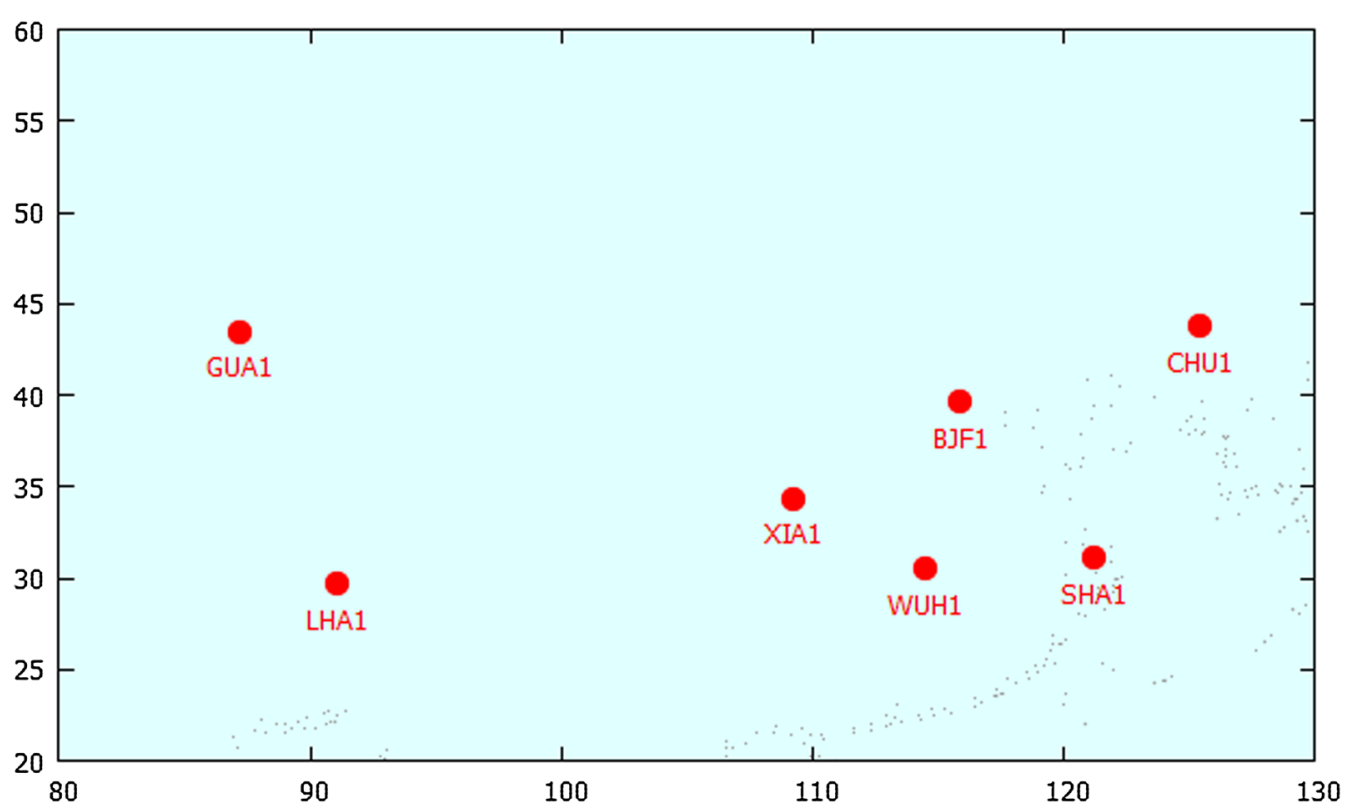

Fig. 1 The distribution of the iGMAS stations. The X-and Y-axis are longitude and latitude in degrees, respectively. The distance between the furthest stations is approximately $1700 \mathrm{~km}$

Table 1 Measurement relationships between satellites or between satellites and anchor stations

\begin{tabular}{|c|c|c|c|c|c|c|c|c|c|c|c|}
\hline & \multicolumn{10}{|c|}{ Signal transmission } \\
\hline & & B19 & B20 & B21 & B22 & B27 & B28 & B29 & B30 & A01 & A02 \\
\hline \multirow{10}{*}{ 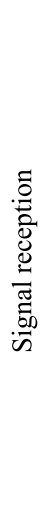 } & B19 & $\lambda$ & & $\sqrt{ }$ & & $\sqrt{ }$ & $\sqrt{ }$ & $\sqrt{ }$ & $\sqrt{ }$ & $\sqrt{ }$ & $\sqrt{ }$ \\
\hline & B20 & & 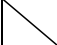 & $\sqrt{ }$ & $\sqrt{ }$ & $\sqrt{ }$ & $\sqrt{ }$ & $\sqrt{ }$ & $\sqrt{ }$ & $\sqrt{ }$ & $\sqrt{ }$ \\
\hline & B21 & $\sqrt{ }$ & $\sqrt{ }$ & 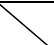 & & $\sqrt{ }$ & $\sqrt{ }$ & $\sqrt{ }$ & $\sqrt{ }$ & $\sqrt{ }$ & $\sqrt{ }$ \\
\hline & B22 & & $\sqrt{ }$ & & 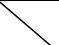 & $\sqrt{ }$ & $\sqrt{ }$ & $\sqrt{ }$ & $\sqrt{ }$ & $\sqrt{ }$ & $\sqrt{ }$ \\
\hline & B27 & $\sqrt{ }$ & $\sqrt{ }$ & $\sqrt{ }$ & $\sqrt{ }$ & 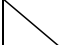 & & $\sqrt{ }$ & & $\sqrt{ }$ & $\sqrt{ }$ \\
\hline & B28 & $\sqrt{ }$ & $\sqrt{ }$ & $\sqrt{ }$ & $\sqrt{ }$ & & 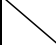 & $\sqrt{ }$ & $\sqrt{ }$ & $\sqrt{ }$ & $\sqrt{ }$ \\
\hline & B29 & $\sqrt{ }$ & $\sqrt{ }$ & $\sqrt{ }$ & $\sqrt{ }$ & $\sqrt{ }$ & $\sqrt{ }$ & & & $\sqrt{ }$ & $\sqrt{ }$ \\
\hline & B30 & $\sqrt{ }$ & $\sqrt{ }$ & $\sqrt{ }$ & $\sqrt{ }$ & & $\sqrt{ }$ & & & $\sqrt{ }$ & $\sqrt{ }$ \\
\hline & A01 & $\sqrt{ }$ & $\sqrt{ }$ & $\sqrt{ }$ & $\sqrt{ }$ & $\sqrt{ }$ & $\sqrt{ }$ & $\sqrt{ }$ & & & \\
\hline & A02 & $\sqrt{ }$ & $\sqrt{ }$ & $\sqrt{ }$ & $\sqrt{ }$ & $\sqrt{ }$ & $\sqrt{ }$ & $\sqrt{ }$ & & & \\
\hline
\end{tabular}

- EXP2: Data from the iGMAS stations and Ka-pseudoranges between satellites were jointly processed with Ka-biases of each satellite estimated simultaneously.

- EXP3: Data from the iGMAS stations and Ka-pseudoranges between satellites were jointly processed with Ka-biases of each satellite calibrated with the mean estimated values obtained in EXP2.

- EXP4: Ka-pseudoranges from satellites and anchor stations were processed with Ka-biases of each satel- lite and anchor station calibrated with the mean estimated values in EXP2 and the estimated values from literature [19], respectively.

Data were processed with the SPODS software [22, 23] on a daily basis with 72-h data started on each day. Time slots of $1 \mathrm{~min}$ were assigned every $5 \mathrm{~min}$ and centered on the sampling epoch, i.e., for the data from monitoring stations, the sampling interval was $5 \mathrm{~min}$. The coordinates of the iGMAS stations, anchor stations, and the earth rotation parameters were fixed to known values. The following orbital dynamics were carefully modeled: gravity of the earth computed with the EGM2008 up to degree and order 12, with linear variation of low-degree coefficients and periodical variation caused by the solid earth tides and the pole tide [24], gravity of the thirdbodies (the moon, sun, and planets) computed with JPL ephemeris DE405, and the general relativistic effects. For each satellite, without any a priori model, the ECOM model [25] was used to model the force acting on the satellites from solar radiation pressure with five parameters $\left(D_{0}, Y_{0}, B_{0}, B_{c}\right.$, and $\left.B_{s}\right)$, estimated together with the initial state vector. The Saastamoinen model [24] was used to calculated the hydrostatic zenith troposphere delay, with wet zenith delay parameter estimated every $2 \mathrm{~h}$ for the monitoring stations and every $24 \mathrm{~h}$ for anchor stations. The Global Mapping Function [26] was adopted to map the troposphere delay from the zenith to the line of sight. The satellite clock drift was replaced with the first order 
coefficient of the broadcast clock correction model. In order to remove the rank defects caused by clock parameters, one clock parameter was chosed as the reference and constrained to 0 in each time slot. Since none of the monitoring stations, anchor stations, and satellites have observation data covering the whole arc, reference clock often changes in different time slots and can be a monitoring station, satellite, or an anchor station, at a certain time slot, depending on their observation number. In EXP2 and EXP5 (see "Orbit determination and time synchronization with single one-way ISL" section), one Ka-bias is chosen as the reference and constrained to 0 because not all Ka-biases are estimable, e.g., the receiving Ka-bias of B21. The pseudoranges and phases of the monitoring stations are weighted according to a prior precisions of $2 \mathrm{~m}$ and $0.02 \mathrm{~m}$, respectively, and the Kapseudorange is weighted according to a prior precision of $0.1 \mathrm{~m}$.

In order to evaluate the accuracy of the obtained orbits and clocks, differences of values in a 48-h overlap arc of adjacent solutions are calculated. The RMS differences for orbits are computed as an indicator of orbit accuracy. Mean value of differences over all satellites are firstly computed and deducted from the original overlapping differences at each epoch; then, the 95th percentile of the resultant differences is an indicator of clock accuracy.

\section{Results}

Figure 2 shows the RMSs in $\mathrm{R}, \mathrm{T}$, and $\mathrm{N}$ directions for each satellite obtained by the four experiments (note that the RMSs of EXP2, EXP3 and EXP4 have a tenfold magnification). It can be seen that in EXP1, the RMSs are larger than $0.3 \mathrm{~m}$ for $\mathrm{R}$, larger than $1 \mathrm{~m}$ for $\mathrm{T}$, and range from 1.2 to $2.3 \mathrm{~m}$ for N. In EXP2, the RMSs are smaller than $0.025 \mathrm{~m}$ in $\mathrm{R}$, are not larger than $0.1 \mathrm{~m}$ for $\mathrm{T}$, and are not larger than $0.15 \mathrm{~m}$ for $\mathrm{N}$. With the Ka-biases of each satellite fixed to the multi-day average estimation in EXP2, the orbit accuracy in EXP3 is further improved. In EXP4, the orbit accuracy in $\mathrm{R}$ is very close to those in EXP2 and EXP3 for each individual satellite. However, the orbit accuracies in $\mathrm{T}$ and $\mathrm{N}$ are worse than those of EXP2 and EXP3, as there are only two anchor stations leading to looser constraints on the constellation orientation.

Figure 3 shows the clock accuracy measured with overlap differences (95\%) for each satellite in the four experiments. It can be seen that in EXP1 the clock accuracy for each satellite is between 1.5 and $2.5 \mathrm{~ns}$. The accuracies for each satellite are better than $0.25 \mathrm{~ns}$ and $0.07 \mathrm{~ns}$ for EXP2 and EXP3, respectively, and the accuracy for each satellite in EXP4 is better than that in EXP3.

Table 2 shows the accuracies of orbits and clocks obtained by different schemes. Compared with EXP1, improvements of orbit accuracies in EXP2 are 95.7\%, 94.5\%, 92.6\%, and $93.4 \%$ in $\mathrm{R}, \mathrm{T}, \mathrm{N}$, and $3 \mathrm{D}$ position, respectively. While, in EXP3, improvements of 95.7\%, 94.5\%, 92.6\%, and 93.4\% are achieved for R, T, N, and 3D position, respectively. In EXP4, the orbit accuracy in the $\mathrm{R}$ direction for each satellite is close to that in EXP3 but less than those of EXP2 and EXP3 in T and N directions. For satellite clocks, compared with EXP1, the accuracies are improved by $90.5 \%$ and $97.2 \%$ in EXP2 and EXP3, respectively. Since the overlap clock differences in EXP2 include the difference of estimated Ka-biases, the nominal accuracy of clocks is less than those in EXP3 and
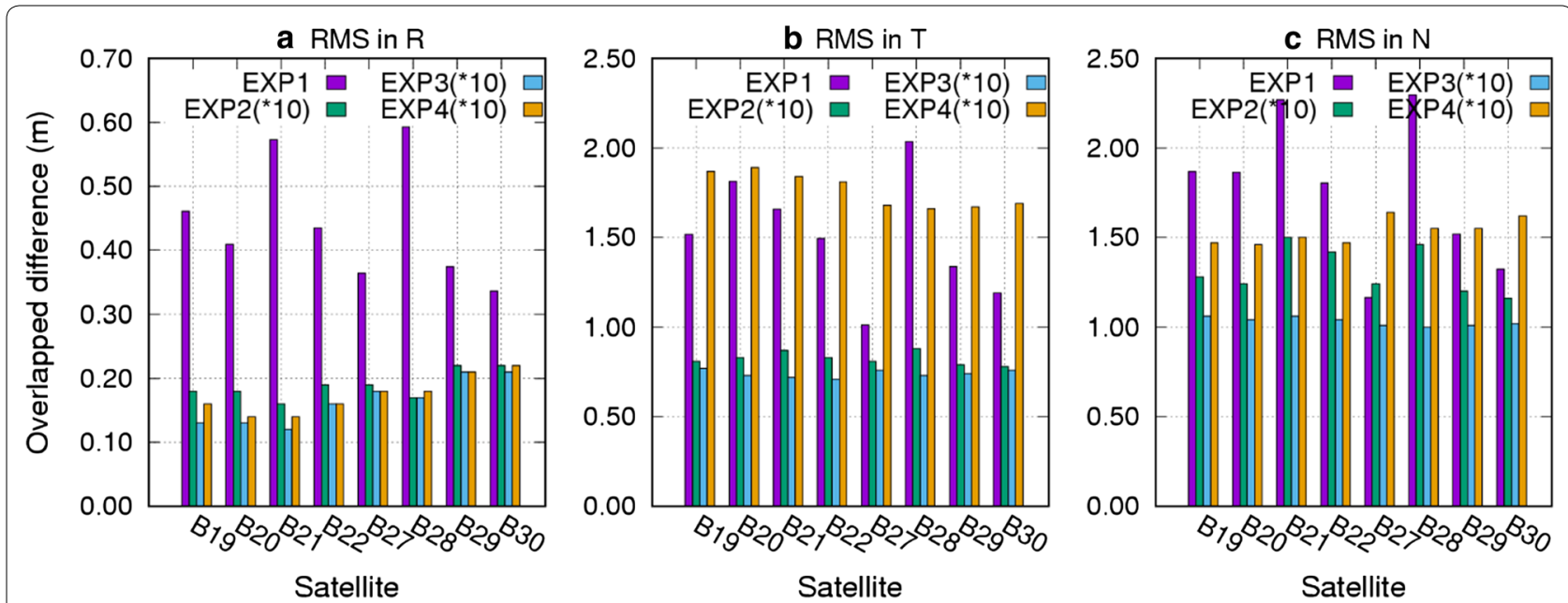

Fig. 2 RMS of overlap orbit differences in $\mathrm{R}, \mathrm{T}$, and $\mathrm{N}$ for each satellite. The values of EXP2, EXP3, and EXP4 have a tenfold magnification to illustrate the differences between them 


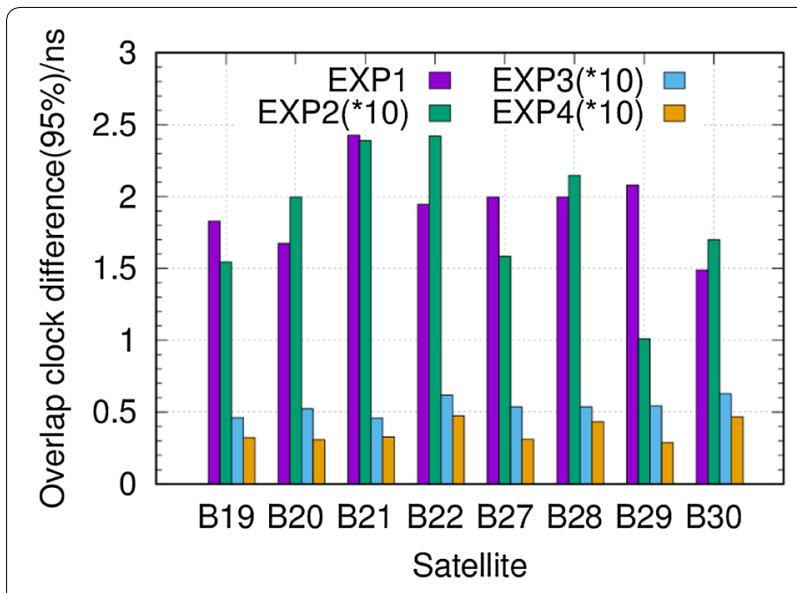

Fig. 3 Overlap clock differences (95\%) in the 4 experiments for each satellite. The values of EXP2, EXP3 and EXP4 have a tenfold magnification to illustrate the differences

Table 2 Accuracies of orbits and clocks obtained by different $O D$ and TS schemes

\begin{tabular}{llllll}
\hline Experiments & \multicolumn{2}{l}{ Orbits $(\mathbf{m})$} & \multirow{2}{*}{ Clocks (ns) } \\
\cline { 2 - 5 } & $\mathbf{R}$ & $\mathbf{T}$ & $\mathbf{N}$ & 3D & \\
\hline EXP1 & 0.443 & 1.507 & 1.763 & 2.364 & 1.929 \\
EXP2 & 0.019 & 0.083 & 0.131 & 0.156 & 0.185 \\
EXP3 & 0.016 & 0.074 & 0.103 & 0.128 & 0.054 \\
EXP4 & 0.017 & 0.176 & 0.153 & 0.235 & 0.037 \\
\hline
\end{tabular}

EXP4. The orbit accuracy in EXP4 is less than those in EXP3 and EXP2 but the clock accuracy is greater, implying model inaccuracies. For example, there may be biases of the antenna phase center for the Ka-band and the L-band, or there may be non-ignorable variation of the hardware delay for the Ka-band and the L-band signal.

Figure 4 shows the variations of estimated receiving and transmitting Ka-biases of each satellite obtained in EXP2 with the bias removed for each individual satellite, so all the values are within the range of the $y$-axis. The Ka-biases of each satellite are stable in time. In Table 3, the standard deviation (SD) of the receiving and transmitting hardware biases for each satellite is smaller than $0.15 \mathrm{~m}$ (or equally $0.5 \mathrm{~ns}$ ).

Figure 5 shows the RMS of residuals for one-way Kapseudoranges observed at each satellite or anchor station in EXP2, EXP3, and EXP4. The RMSs of all satellites are smaller than $0.08 \mathrm{~m}$; the RMSs for satellite B27-B30 are larger than those of other satellites, and satellite B28 has the largest RMS. The RMSs of individual satellites from different experiments are very close to each other. In EXP2, the average RMS is $0.054 \mathrm{~m}$ for all satellites, while it is $0.040 \mathrm{~m}$ for $\mathrm{B} 19-\mathrm{B} 22$ and $0.067 \mathrm{~m}$ for $\mathrm{B} 27-\mathrm{B} 28$. It can be seen that the RMSs of the two anchor stations are larger than those of the satellites. It is noticed that the RMSs for satellite B27-B30 in EXP2 and EXP3 are larger than those in EXP4, which may also be due to incorrect assumptions for the stability of hardware delays.

Figure 6 shows the percentage of valid one-way Kapseudorange data in EXP3 and EXP4 for each satellite or anchor station. The percentage of valid data received or transmitted at satellites and anchor stations is more than $97 \%$ and the percentage of valid data received at B28 is the lowest. In EXP4, the percentage for each satellite is larger than that in EXP3. The average percentage for all satellites is $98.82 \%$ in EXP3 and $99.55 \%$ in EXP4. This also confirms that there are possible modeling errors, when the Ka-band data and the L-band monitoring data are jointly processed.

\section{Orbit determination and time synchronization with single one-way ISL}

Compared to the existing methods, only single one-way ranging data is necessary for OD and TS in the proposed method. In order to prove this point, EXP5, EXP6, and EXP7 were performed with the same data processing strategies as used in EXP2, EXP3, and EXP4, but only single one-way Ka-pseudorange data from measurement links were used (denoted by the red " $\sqrt{ }$ " in Table 1).

The accuracies of orbits for each satellite in EXP5, EXP6, and EXP7 are shown in Fig. 7. We can see that, the accuracies of orbits in each component in all experiments are all greater than $0.05 \mathrm{~m}$. As expected, the accuracies in EXP6 are slightly higher than those in EXP5, and the accuracies are greater than $0.04 \mathrm{~m}, 0.1 \mathrm{~m}$, and $0.2 \mathrm{~m}$ in $\mathrm{R}$, $\mathrm{T}$, and $\mathrm{N}$, respectively, for both experiments. In EXP7, the orbit accuracy in R is similar to those in EXP5 and EXP6, but the accuracies in $\mathrm{T}$ and $\mathrm{N}$ are about $0.3 \mathrm{~m}$ and much worse than those in EXP5 and EXP6, for the same reason mentioned above.

Figure 8 compares the clock accuracies obtained by different processing modes under the condition of single or double one-way Ka-pseudoranges. It can be seen that in EXP5, the clock accuracies of different satellites are between 0.2 and $0.35 \mathrm{~ns}$, and the clock accuracies obtained by EXP6 and EXP7 are similar, and all of them are no more than $0.2 \mathrm{~ns}$.

Table 4 shows the accuracies for orbits and clocks obtained by EXP5, EXP6, and EXP7. It can be seen that the orbit accuracy in $\mathrm{R}$ is greater than $0.027 \mathrm{~m}$ in the three experiments, and the accuracies are decreased by up to $60 \%$ compared with those obtained with double one-way data. In other words, the radial orbital accuracies are improved by up to $40 \%$ using double one-way data. The clock accuracies obtained by the three experiments are 

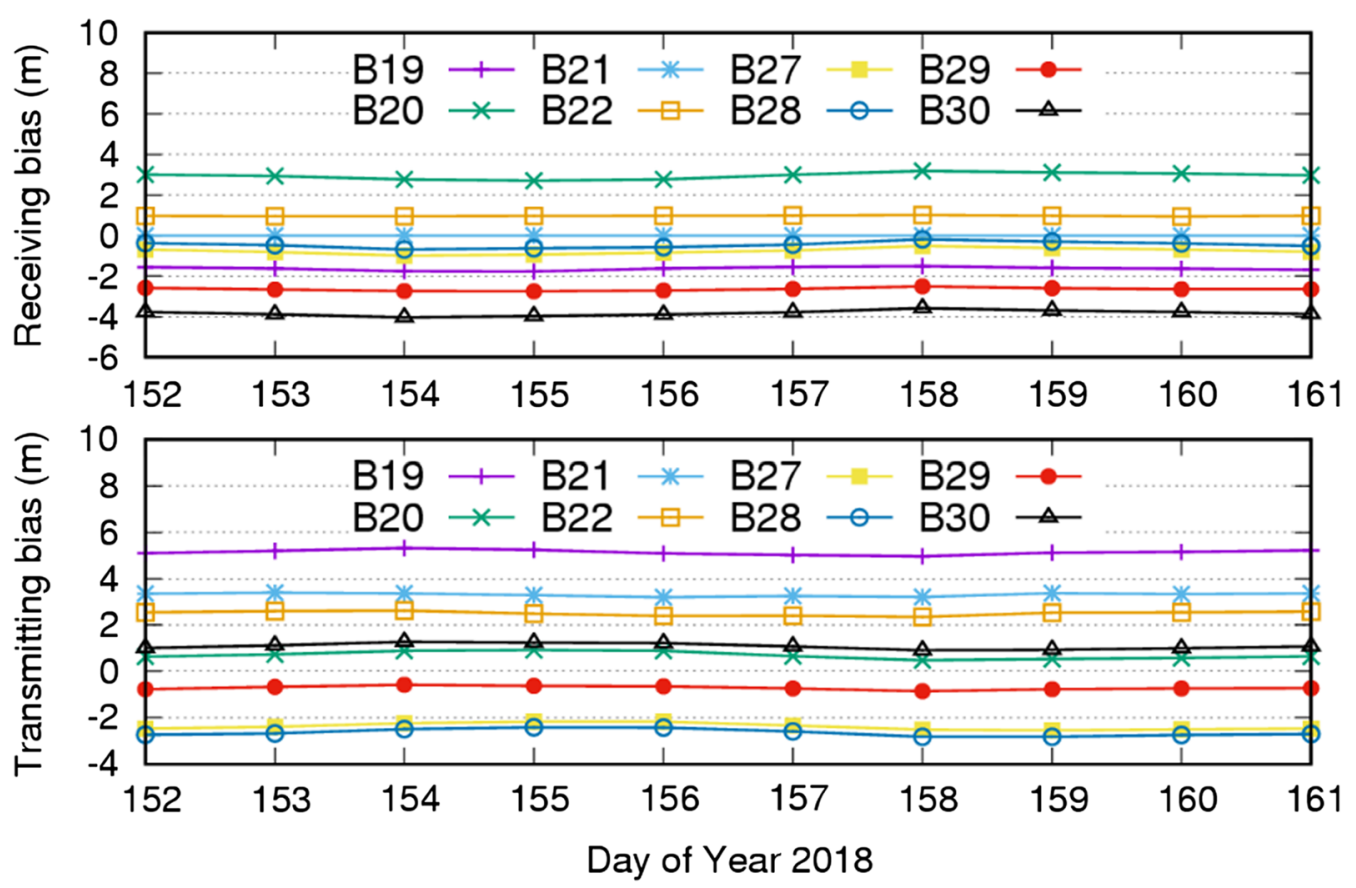

Fig. 4 Variations of estimated receiving and transmitting Ka-biases for ISL equipment of each satellite obtained in EXP2. A large bias has been removed for each individual satellite, so all the values of all satellites are within the range of the $y$-axis

Table 3 Repeatability of the estimated Ka-biases for each satellite obtained in EXP2 (unit: $\mathrm{m}$ )

\begin{tabular}{lllllllll}
\hline Satellite & B19 & B20 & B21 & B22 & B27 & B28 & B29 & B30 \\
\hline Receiving Ka-bias & 0.083 & 0.150 & - & 0.021 & 0.139 & 0.145 & 0.070 & 0.128 \\
Transmitting Ka-bias & 0.102 & 0.149 & 0.068 & 0.091 & 0.137 & 0.147 & 0.075 & 0.119 \\
\hline
\end{tabular}

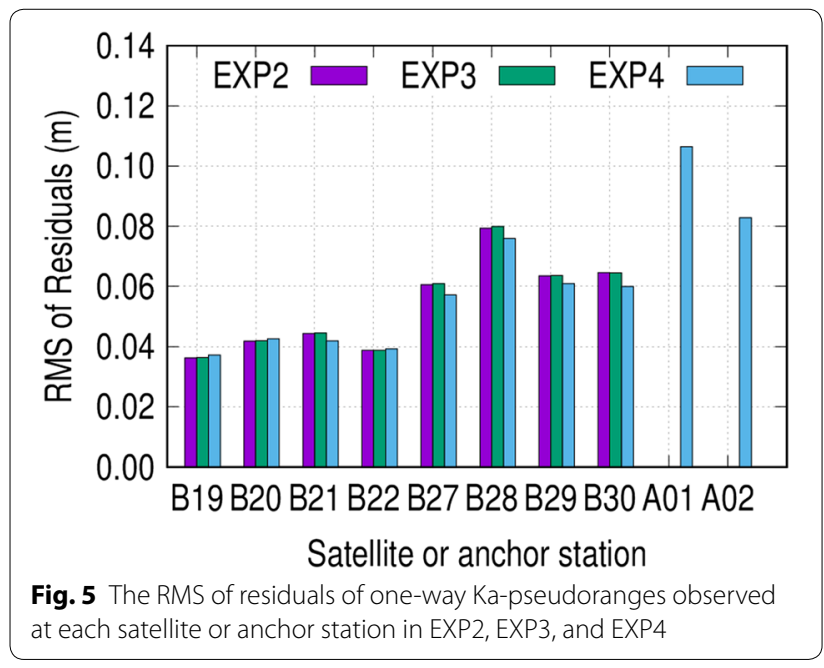

greater than $0.3 \mathrm{~ns}$ and greater than $0.16 \mathrm{~ns}$ in EXP6 and EXP7. Compared with the results obtained with double one-way data as shown in Table 3, the clock accuracies decreased by $42 \%, 183 \%$, and $297 \%$ in EXP5, EXP6, and EXP7, respectively. This implies that the clocks benefit more from double one-way data than the orbits do.

Table 5 provides the repeatability of the estimated Kabiases for each satellite obtained by EXP5 and the differences between the mean estimated Ka-biases obtained by EXP5 and those obtained by EXP2. The SDs of the obtained receiving biases in EXP5 do no exceed $0.18 \mathrm{~m}$ and the differences compared with those in EXP2 are less than $0.082 \mathrm{~m}$. For the transmitting biases, the SDs are smaller than $0.14 \mathrm{~m}$ while the differences do not exceed $0.04 \mathrm{~m}$. 


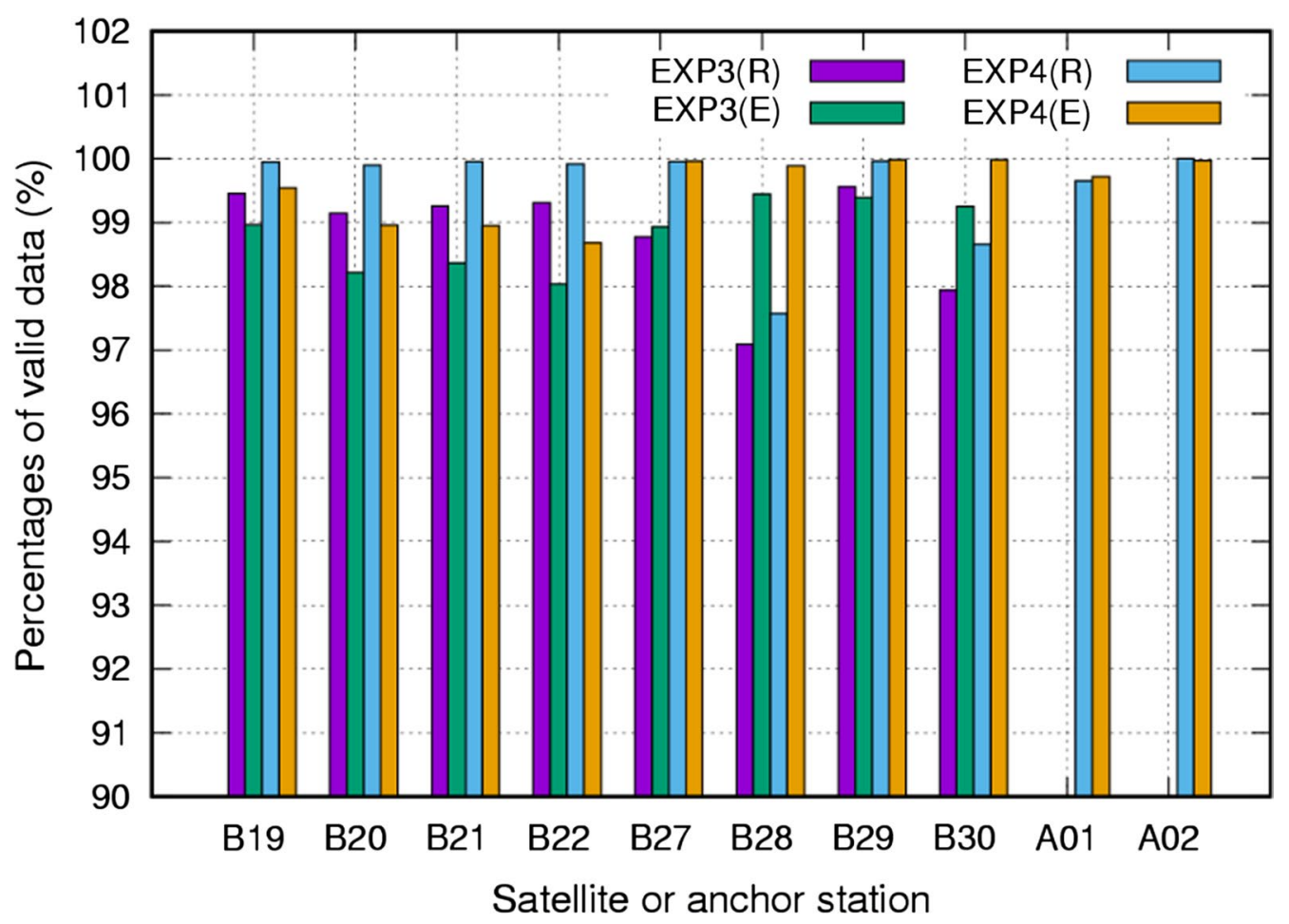

Fig. 6 The percentage of valid one-way Ka-pseudorange data in EXP3 and EXP4 for each satellite or anchor station. R and E in the legend represent reception and transmission, respectively

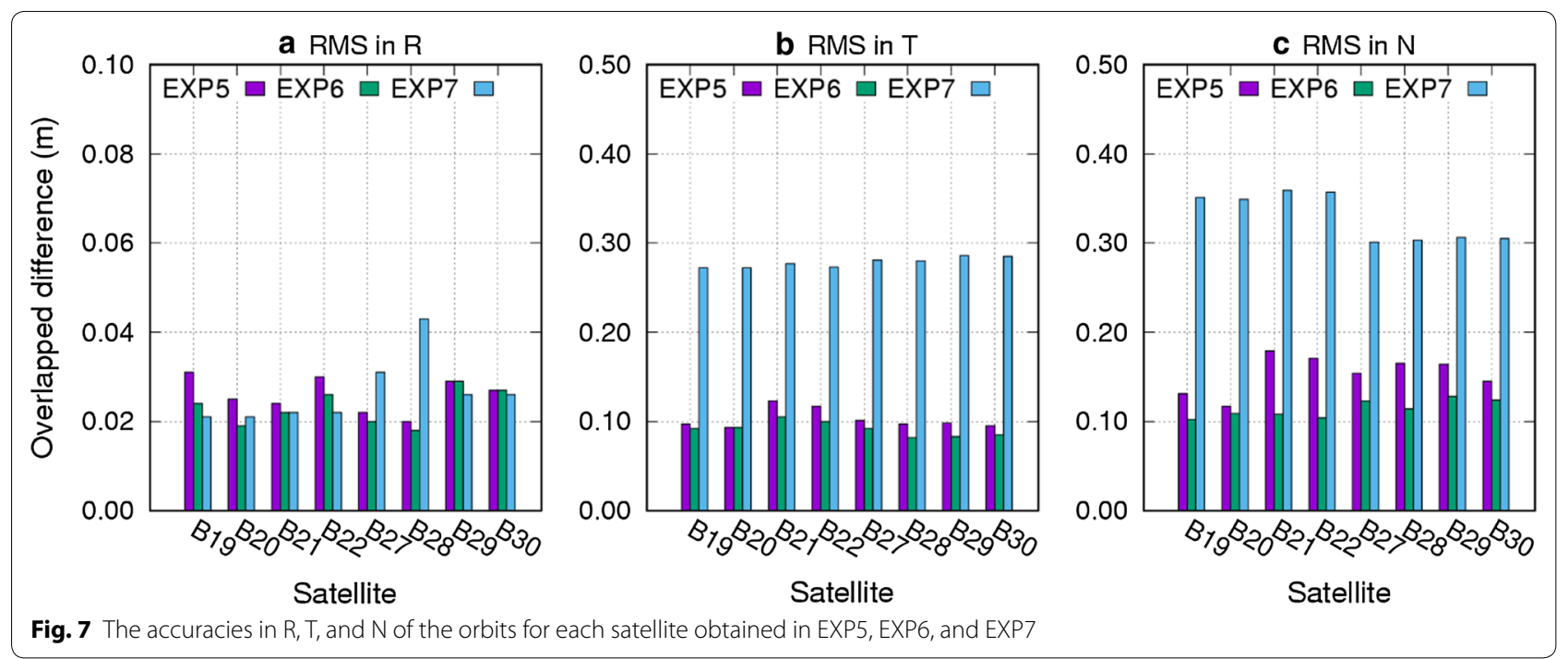

\section{Discussion}

Since the ISL of BDS-3 runs in a TDMA mode, the Kapseudorange observations at a certain satellite are generated at different times, and each observation contains two unique clock offset parameters that remove redundant observations. Existing methods for OD and TS with
ISL data firstly derive the raw Ka-pseudorange observations from different times to some specified time epoch, and then the DCMs and the DEMs are formed to separate the orbit and clock information so that OD and TS are separated [17]. This idea has been first proposed by Ananda et al. [8] and applied to OD and TS for BDS-3 


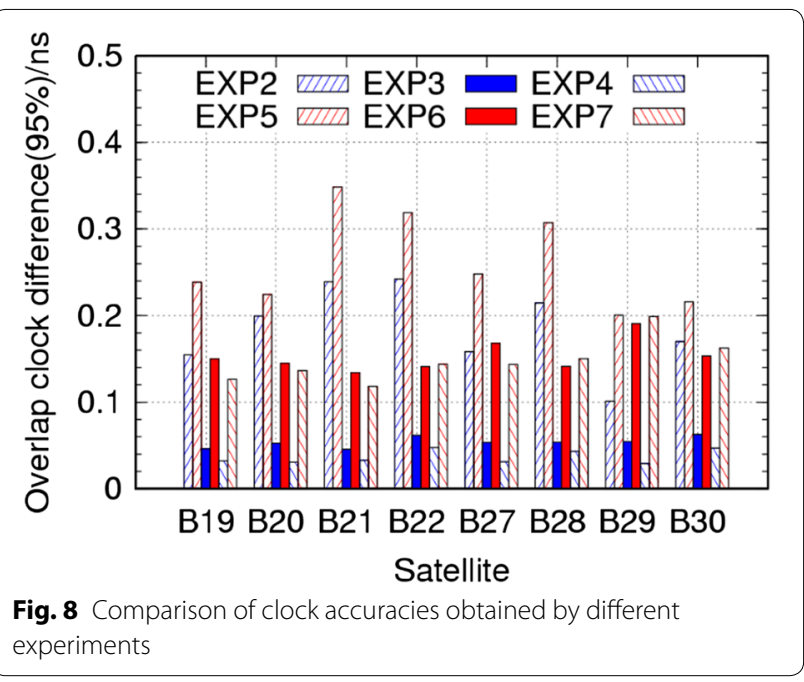

Table 4 Accuracies for orbits and clocks obtained by different $O D$ and TS schemes under the condition of single one-way Ka-pseudoranges

\begin{tabular}{llllll}
\hline \multirow{2}{*}{ Experiments } & \multicolumn{2}{l}{ Orbits $(\mathbf{m})$} & \multirow{2}{*}{ Clocks (ns) } \\
\cline { 2 - 4 } & $\mathbf{R}$ & $\mathbf{T}$ & $\mathbf{N}$ & 3D & \\
\hline EXP5 & 0.026 & 0.103 & 0.153 & 0.186 & 0.263 \\
EXP6 & 0.023 & 0.092 & 0.114 & 0.149 & 0.153 \\
EXP7 & 0.027 & 0.278 & 0.329 & 0.432 & 0.147 \\
\hline
\end{tabular}

satellites in the operational control center $[2-4,6]$. It may have advantages in stability and computational efficiency for on-board distributed autonomous OD and TS, but for routine OD and TS at the operational control center or for the centralized autonomous OD and TS, its shortcomings are obvious. For example, data processing with this method is cumbersome, and it is difficult to calibrate the hardware delay of ISL equipment. To compute DEMs and DCMs, if either of the required double oneway pseudorange observations is not present (e.g., satellite observations do not arrive in time) or lacks quality, then the other will be discarded. If the method of polynomial interpolation or fitting is employed to compute derived pseudorange observations for a certain satellitepair, a sequence of one-way pseudorange observations with a certain length is needed for each derived observation, and the interval of the sequence must contain the "observing" time of the required derived observation.

The method proposed in this paper directly uses the raw ISL pseudoranges for OD and TS simultaneously, and does not require either to generate derived observation or to match one-way pseudoranges observed at satellite-pairs. The procedure of data processing is very simple, almost the same as that for processing observation monitoring station data. Compared with the existing methods, the greatest advantage of this method is that it can simultaneously determine satellite orbits and clocks and the hardware delay biases of ISL equipment on satellites, if observations from monitoring stations are jointly processed. The obtained orbits and clocks are more selfconsistent, and the estimated ISL hardware biases can be used to correct the ISL observation data for autonomous OD and TS. Another advantage of directly processing raw one-way pseudorange data is that data quality or performance of each ISL transmitting or receiving device at each satellite can be analyzed independently.

The proposed method for OD and TS requires only single one-way pseudorange data, so there is no need to wait for the return of the data observed at those satellites that are beyond tracking by the ground stations, increasing the data availability. Only $20 \%$ of all the ISL data were used for validation (the length of time slots was $1 \mathrm{~min}$ and the sampling interval was $5 \mathrm{~min}$ ). This implies that the frequency for ranging or transferring ISL data can be reduced to allow more time for inter-satellite communication or more channel resources for transferring other information.

\section{Conclusions}

For OD and TS of BDS-3 satellites with the ISL operated in a TDMA mode, a general model using simultaneous or non-simultaneous observation data is proposed. The proposed approach was validated with experiments for two representative and important modes of OD and TS:

Table 5 Repeatability of the estimated Ka-biases for each satellite obtained by EXP5 and the differences (denoted by $\nabla$ ) between the mean estimated Ka-biases obtained by EXP5 and those obtained by EXP2

\begin{tabular}{|c|c|c|c|c|c|c|c|c|}
\hline Satellite & B19 & B20 & B21 & B22 & B27 & B28 & B29 & B30 \\
\hline \multicolumn{9}{|l|}{ Receiving } \\
\hline$\nabla$ & -0.012 & 0.027 & - & -0.013 & -0.082 & -0.071 & - & - \\
\hline STD & 0.105 & 0.161 & - & 0.033 & 0.167 & 0.173 & - & - \\
\hline \multicolumn{9}{|c|}{ Transmitting } \\
\hline$\nabla$ & - & - & 0.025 & 0.022 & 0.016 & 0.021 & -0.032 & -0.020 \\
\hline STD & - & - & 0.099 & 0.126 & 0.126 & 0.137 & 0.108 & 0.131 \\
\hline
\end{tabular}


(1) jointly processing ISL Ka-pseudoranges between satellites and ionosphere-free pseudorange and phase data from monitoring stations, and (2) jointly processing Kapseudoranges between satellites and between satellites and anchor stations. The results show that by jointly processing ISL data and the data of seven iGMAS stations located in China, the average RMS overlap orbit difference of the eight BDS-3 satellites is $0.019 \mathrm{~m}$ for radial direction and $0.156 \mathrm{~m}$ for 3D position; the overlap clock differences (95\%) are $0.185 \mathrm{~ns}$, and the standard deviation of the estimated Ka-biases is greater than 0.5 ns. Compared with the results obtained with only the seven monitoring stations, the improvements for orbits in radial and clocks are $95.7 \%$ and $90.5 \%$, respectively. If the Ka-biases are fixed to known (pre-estimated) values, the accuracies of orbits and clocks are further improved; e.g., the overlap clock difference (95\%) reduces to 0.054 ns. By jointly processing Ka-pseudoranges observed by satellites and two anchor stations, the average RMSs of overlap orbit differences are $0.017 \mathrm{~m}$ in the radial direction and $0.235 \mathrm{~m}$ in the 3D position, while the overlap clock difference (95\%) is $0.037 \mathrm{~ns}$. It is also shown that under the condition of single one-way ranging link, the accuracy of orbits and clocks obtained by the two modes is still significantly better than that obtained by only using data from monitoring stations. The RMS orbit difference is no more than $0.03 \mathrm{~m}$ in $\mathrm{R}$, the overlap clock difference (95\%) is no more than $0.3 \mathrm{~ns}$, and the SDs of estimated Ka-biases are less than $0.6 \mathrm{~ns}$.

As shown in the paper, the accuracy of clocks obtained by processing Ka-pseudoranges observed at anchor stations and satellites is greater than that obtained by jointly processing ISL data and monitoring station data. This may imply that not all error is modeled correctly; this could be due to the phase center offsets for the L-band and Ka-band signal or the time variation of hardware delay in the L-band and Ka-band equipment.

\section{Acknowledgements \\ Thanks to the International GNSS Monitoring and Assessment System for providing the monitoring station data.}

\section{Authors' contributions}

$X J, L F$, and ZW evaluated the accuracy of the clock solutions. JZ, ZH, and JL evaluated the accuracy of orbit solutions. All authors read and approved the final manuscript.

\section{Funding}

This study was supported by the National Natural Science Foundation of China (Grant Nos. 41704035 and 42774012) and Office of China Navigation Satellite System (Project No. GFZX03010403).

\section{Availability of data and material}

The data analyzed during the current study are not publicly available due to relevant laws of China.

Ethics approval and consent to participate Not applicable.

\section{Competing interests}

The authors declare that they have no competing interests.

\section{Author details}

${ }^{1}$ Xi'an Research Institute of Surveying and Mapping, Xi'an 710054, China.

${ }^{2}$ State Key Laboratory of Geo-information Engineering, Xi'an 710054, China.

${ }^{3}$ Information Engineering University, Zhengzhou 450001, China. ${ }^{4}$ China Xi'an Satellite Control Center, Xi'an 710043, China. ${ }^{5}$ State Key Laboratory of Astronautic Dynamics, Xi'an 710043, China.

Received: 1 July 2019 Accepted: 7 January 2020

Published online: 02 March 2020

\section{References}

1. Yang, Y., Xu, Y., Li, J., \& Yang, C. (2018). Progress and performance evaluation of BeiDou global navigation satellite system: Data analysis based on BDS-3 demonstration system. Sci China Earth Sci., 61(5), 614-624.

2. Tang, C., Hu, X., Zhou, S., \& Liu, L. (2018). Initial results of centralized autonomous orbit determination of the new-generation BDS satellites with inter-satellite link measurements. Journal of Geodesy, 92(10), 1155-1169.

3. Yang, D., Yang, J., Li, G., Zhou, Y., \& Tang, C. (2017). Globalization highlight: Orbit determination using BeiDou inter-satellite ranging measurements. GPS Solutions, 21(3), 1395-1404. https://doi.org/10.1007/s1029 1-017-0626-5.

4. Pan, J., Hu, X., Zhou, S., Tang, C., Guo, R., Zhu, L., et al. (2018). Time synchronization of new-generation BDS satellites using inter-satellite link measurements. Advances in Space Research, 61(1), 145-153. https://doi. org/10.1016/j.asr.2017.10.004.

5. Song, X., Mao, Y., Feng, L., Jia, X., \& Ji, J. (2017). The preliminary result and analysis for BD orbit determination with inter-satellite link data. Acta Geodaetica et Cartographica Sinica, 46(5), 547-553.

6. Ren, X. (2018). Research on the theory and method of BDS autonomous orbit determination. Ph.D. Information Engineering University, Zhengzhou.

7. Ren, X., Yang, Y., \& Zhu, J. (2017). Orbit determination of the Next-Generation BeiDou satellites with Inter-satellite link measurements and a priori orbit constraints. Advances in Space Research, 60(10), 2155-2165. https:// doi.org/10.1016/j.asr.2017.08.024.

8. Ananda, M. P., Bernstein, H., Cunningham, K. E., Feess, W. A., \& Stroud, E. G. (1990). Global positioning system (GPS) autonomous navigation. Paper presented at the IEEE position location and navigation symposium.

9. Lin, Y., Qin, Z., Chu, H., \&Wang, H. (2010). A satellite cross link-based GNSS distributed autonomous orbit determination algorithm. Journal of Astronautics, 2010(31), 2088-2094.

10. Zhu, J. (2011). Research on orbit determination and time synchronizing of navigation satellite based on crosslinks. Ph.D., National University of Defense Technology, Changsha.

11. Mao, Y., Song, X., Jia, X., \& Wu, X. (2013). Naturalisation method research on inter-satellite link observation data. Geomatics and Information Science of Wuhan University, 38(9), 1201-1206.

12. Song, X. (2009). Study on the orbit determination of COMPASS navigation satellites. Ph.D., Chang'an University, Xi'an.

13. Hein, G. W. (2011). Inter satellite links for GNSS. Presentation at University of FAF Munich, Xi'an.

14. Fernández, F. A. (2011). Inter-satellite ranging and inter-satellite communication links for enhancing GNSS satellite broadcast navigation data. Advances in Space Research, 47(5), 786-801.

15. Chen, J., You, Z., \& Jiao, W. (2005). Research on autonav of navigation satellite constellation based on crosslink range and inter-satellite orientation observation. Journal of Astronautics, 26(1), 43-46.

16. Ruan, R., Feng, L., Jia, X., Wu, X., Song, X., \& He, T. (2012). Estimation of crosslink equipment delay and its contribution to GNSS orbit determination and time synchronization. Paper presented at the China Satellite Navigation Conference(CSNC) 2012 Proceedings (Vol. 160) Guangzhou.

17. Ruan, R., Feng, L., \& Jia, X. (2014). Equipment delay estimation for GNSS satellite combined orbit determination with satellite-ground link and inter-satellite link observations. Acta Geodaetica et Cartographica Sinica, 43(02), 137-142, 157. 
18. Liu, W., Gong, X., \& Zhang, W. (2011). GPS orbit determination with real ground tracking observations and simulative crosslink ranging observations. Paper presented at the CSNC 2011, Shanghai.

19. Ruan, R. (2018). Research on key technologies of precise data processing for GNSS networks. Ph.D., Information Engineering University, Zhengzhou.

20. Ruan, R., Wei, Z., \& Jia, X. (2019). BDS-3 satellite orbit and clock determination with one-way inter-satellite pseudorange and monitoring station data. Acta Geodaetica et Cartographica Sinica, 48(3), 267-275.

21. Ruan, R., \&Wei, Z. (2019). Between-satellite single-difference integer ambiguity resolution in GPS/GNSS network solutions. Journal of Geodesy.. https://doi.org/10.1007/s00190-019-01251-Z.

22. Wei, Z., Ruan, R., Jia, X., Wu, X., Song, X., Mao, Y., et al. (2014). Satellite positioning and orbit determination system SPODS: Theory and test. Acta Geodaetica et Cartographica Sinica, 43(1), 1-4.

23. Ruan, R., Jia, X., Wu, X., Feng, L., \& Zhu, Y. (2014). SPODS software and its result of precise orbit determination for GNSS satellites. Paper presented at the China Satellite Navigation Conference (CSNC) 2014 Proceedings (Vol. III) Nanjing.
24. McCarthy, D. D., \& Petit, G. (2004). IERS conventions (2003). International Earth Rotation and Reference Systems Service (IERS).

25. Springer, T. A. (1999). Modeling and validating orbits and clocks using the global positioning system. Phi, Astronomical Institute, University of Berne, Berne, Switzerland.

26. Beohm, J., Neill, A., Tregoning, P., \& Schuh, H. (2006). Global Mapping Function (GMF): A new empirical mapping function based on numerical weather model data. Geophysical Research Letters, 33, L07304.

\section{Publisher's Note}

Springer Nature remains neutral with regard to jurisdictional claims in published maps and institutional affiliations.

\section{Submit your manuscript to a SpringerOpen ${ }^{\odot}$ journal and benefit from:}

- Convenient online submission

- Rigorous peer review

- Open access: articles freely available online

- High visibility within the field

- Retaining the copyright to your article

Submit your next manuscript at $\boldsymbol{\nabla}$ springeropen.com 